\title{
Microwave endometrial ablation as an alternative to hysterectomy for the emergent control of uterine bleeding in patients who are poor surgical candidates
}

\author{
Shamima Yeasmin · Kentaro Nakayama • Masako Ishibashi • Atsuko Katagiri • \\ Kouji Iida $\cdot$ Naomi Nakayama $\cdot$ Showa Aoki $\cdot$ Yasushi Kanaoka $\cdot$ Kohji Miyazaki
}

Received: 10 August 2008 / Accepted: 2 December 2008 / Published online: 19 December 2008

(C) The Author(s) 2008. This article is published with open access at Springerlink.com

\begin{abstract}
Background Microwave endometrial ablation is a new, minimally invasive treatment option for menorrhagia. Its popularity in many countries is increasing due to its safety and simplicity.

Cases We treated menorrhagia due to submucosal myomas in two patients with a modified microwave endometrial ablation device. Surgery was contraindicated in the first patient secondary to medical co-morbidities and in the second patient because of acute hemorrhagic shock. In both cases, the operation was highly effective and each patient was satisfied with her treatment outcome.

Conclusion Given its safety, simplicity, and effectiveness, microwave endometrial ablation may be widely adopted for the emergent control of uterine bleeding in patients with poor surgical candidates.
\end{abstract}

Keywords MEA $\cdot$ Menorrhagia $\cdot$ Multiple systemic diseases $\cdot$ Acute hemorrhagic shock

S. Yeasmin $\cdot$ K. Nakayama $(\bowtie) \cdot$ M. Ishibashi .

A. Katagiri - K. Iida $\cdot$ N. Nakayama $\cdot$ S. Aoki $\cdot$ K. Miyazaki

Department of Obstetrics and Gynecology,

Shimane University School of Medicine,

Enyacho 89-1, Izumo, Shimane 6938501, Japan

e-mail:kn88@med.shimane-u.ac.jp

Y. Kanaoka

Department of Obstetrics and Gynecology,

Osaka City University Graduate School of Medicine,

Osaka City, Osaka, Japan

\begin{abstract}
Abbreviations
MEA Microwave endometrial ablation
\end{abstract}

\section{Introduction}

Endometrial ablation (EA) is a minimally invasive surgical treatment for menorrhagia. Microwave endometrial ablation (MEA) is a second-generation method of EA. It utilizes microwaves at a fixed frequency, delivered by inserting a microwave probe into the uterine cavity. The microwaves destroy the basal layer of the endometrium and the glands by heating them to over $60^{\circ} \mathrm{C}$. The remainder of the uterus is spared. MEA was introduced in 1995 and was found to be an effective, less invasive alternative to hysterectomy [1]. The procedure was subsequently modified to make it safer and easier. MEA has several advantages over other treatments of menorrhagia including hysterectomy and uterine artery embolization. It is quick and easy to perform, requiring minimal operative skill, and has few complications. Preoperative preparation is straightforward and the procedure can be done at a day surgery center. Thus, MEA is relatively cost effective [2]. Recently, a number of cases documenting successful treatment of menorrhagia with MEA in patients with medical complications have been reported $[2,3]$. We report two additional cases of heavy uterine bleeding caused by submucous myomas successfully controlled by MEA. One of the patients had multiple systemic disorders rendering her a poor surgical candidate. The other patient was treated with MEA emergently in order to control uterine bleeding that was leading to hemorrhagic shock. Neither patient had intra- or postoperative complications. At follow-up, both patients experienced complete resolution of menorrhagia with a dramatic reduction in uterine size. 


\section{Materials and methods}

Specific case details are discussed subsequently. Both patients were perimenopausal and had contraindications to hysterectomy. We confirmed the site, size, and vascularity of submucosal myomas by magnetic resonance imaging (MRI) and transvaginal ultrasound. Endometrial malignancy was ruled out by diagnostic imaging (MRI and TVS) and cytological examinations. Written informed consents were obtained from both patients. Permission to perform the MEA was obtained from the ethical committee of the Shimane University hospital. MEA was performed under spinal anesthesia using a device manufactured by Alfresapharma (Osaka, Japan). The device consists of a Sounding Applicator and a microwave generator. The applicator is only $4 \mathrm{~mm}$ in diameter and $20 \mathrm{~cm}$ in length, terminating at the tip with a curved microwave applicator. The $2.45-\mathrm{GHz}$ microwaves are supplied by a Microtaze AZM-520 microwave generator. It is in contrast to a previous microwave device which is $8.5 \mathrm{~mm}$ in diameter and has a non-bending tip and can generate microwaves at $9.2 \mathrm{GHz}$ [1]. This modified shape facilitates the easier and safer operation of this device, even in a large and distorted uterine cavity [4]. For each irradiation site, microwaves were transmitted at $70 \mathrm{~W}$ for approximately $50 \mathrm{~s}$. Neither patient had any intra- or post-operative complications. Both the patients had post-operative visits at 1 week and at 1-3 months that included a careful interview regarding uterine bleeding and clinical examination. MRI images after MEA showed a gradual reduction in the size of the myomas and uterus. Gadolinium (Gd) enhanced MRI demonstrated the necrotic endometrium as an avascular area.

\section{Case 1}

The patient was a 40-year-old woman with history of severe hypertension, cerebral stroke, and atrial fibrillation who presented with severe menorrhagia since several months before operation. On examination, she was found to be anemic. Abdominal examination revealed a markedly enlarged uterus. Transvaginal ultrasonography demonstrated a myoma measuring $5 \mathrm{~cm} \times 4 \mathrm{~cm} \times 5 \mathrm{~cm}$, located on posterior wall. Although the patient was parous, she was a poor candidate for hysterectomy under general anesthesia. Thus, we opted for MEA under the guidance of transabdominal ultrasonography. The patient was not pretreated with either gonadotropin-releasing hormone $(\mathrm{Gn}-\mathrm{RH})$ analogues or danazol for endometrial preparation; MEA was performed under spinal anesthesia. The total operating time was $21 \mathrm{~min}$ and the estimated blood loss was of insufficient volume to record. There were no post-operative complications. An MRI was performed at 7 days, 1 month, and 3 months after MEA (Fig. 1). The MRI on the first week after MEA showed almost no change of myoma size, but there was a small area of avascularity in the endometrium on the posterior wall close the myoma. A Gd-enhnaced T1 MRI at 3 months after treatment shows that myoma size was reduced remarkably and the uterine lining was partially replaced by avascular area (Fig. 1). For case 1, a Gdenhanced T1-weighted image 3 months after the MEA revealed that the intramural myoma on the posterior wall had shrunk to $3 \mathrm{~cm} \times 2 \mathrm{~cm} \times 3 \mathrm{~cm}$. The patient resumed her regular menstrual cycle the next month with underaverage flow.

\section{Case 2}

The patient, a 51-year-old woman, presented to the emergency department with heavy vaginal bleeding leading to hemorrhagic shock. The transvaginal ultrasonography showed a submucosal myoma measuring $2 \mathrm{~cm} \times 3 \mathrm{~cm} \times$ $2 \mathrm{~cm}$. After initial resuscitation, we opted to ablate the bleeding area using microwaves. Under spinal anesthesia,

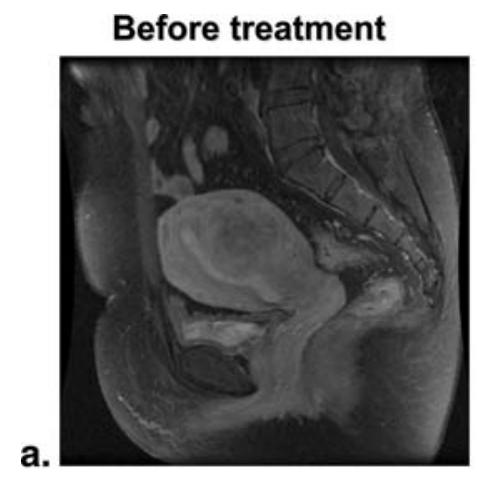

T1WI Gd

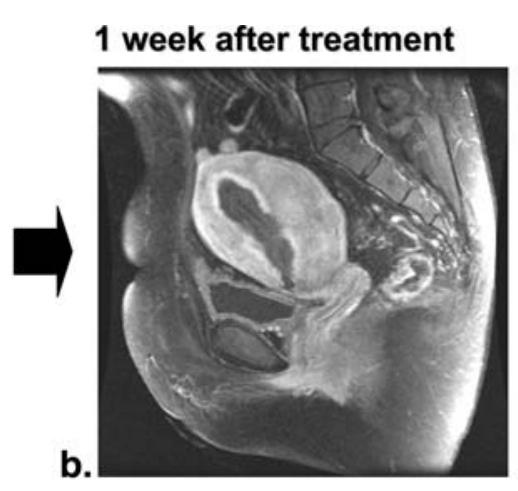

T1WI Gd

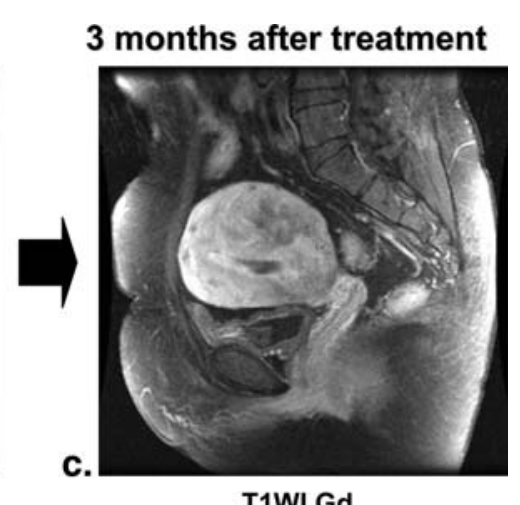

T1WI Gd
Fig. 1 MRI images of case 1. a Axial and T1-weighted images before MEA. The uterine cavity is enlarged and distorted by a submucous myoma, b and c Gd-enhanced T1-weighted images 7 days and
3 months after MEA showing a partially necrotic endometrium. The myoma is smaller than before the MEA 
MEA was performed. The total operative time was $30 \mathrm{~min}$, the estimated blood loss was $90 \mathrm{ml}$, and there were no intra- or post-operative complications. MRI was performed at 5 days, and 3 months post-operatively (Fig. 2). The 3months post-operative MRI revealed that the submucosal myoma on the anterior wall had shrunk to the extent that it became almost undetectable and the uterine lining was partially replaced by an avascular area (Fig. 2). On follow-up, the patient was satisfied with the treatment. She resumed regular menstrual cycles with normal blood loss at 60 days post-operative.

\section{Discussion}

Menorrhagia and metrorrhagia are two of the most frequently reported gynecologic concerns. The search is ongoing for new treatments for abnormal uterine bleeding. Many of the current medical therapies have side effects that limit their duration of use. Although hysterectomy is curative for menorrhagia, there is significant morbidity and mortality associated with the operation. In an attempt to reduce hysterectomy rates and the rates of its associated complications, minimally invasive procedures including uterine artery embolization, hysteroscopic myomectomy, focused ultrasound, and cryomyolysis have been developed. Unfortunately, each of these alternatives has a risk of its own. These procedures may result in any of the following complications, such as: fluid overload, uterine perforation, infection, thermal injury, hemorrhage, deep venous thrombosis, etc. In addition, these procedures can be technically difficult for general practitioners to perform. Recently, efforts have been directed to develop new techniques for destroying the endometrium that are safer and simpler to perform. The new devices ablate the endometrium with lasers, radiofrequency waves, electrocautery, microwaves, heated saline, or a heated balloon. These procedures can be performed with minimal surgical skill in patients who would otherwise be poor surgical candidates. Blood loss is minimal and complications are few. The short-term curative rate of MEA is similar to that of a total hysterectomy. In terms of operative complications and post-operative recovery, MEA is obviously superior to total hysterectomy. It can also be used to treat larger or irregular cavities [4], and has even been used successfully in a didelphic uterus [5]. MEA is only contraindicated when the uterine bleeding arises from a malignancy. There is, however, one case report documenting successful MEA without recurrence for endometrial hyperplasia with atypia. An increasing number of reproductive-aged women are choosing endometrial
Fig. 2 MRI images of case 2, 5 days and 3 months after MEA. a, b T2-weighted images show gradual shrinkage of the myoma and c, d Gd-enhanced T1weighted images show the partially necrotic endometrium following MEA

\section{5 days after treatment}

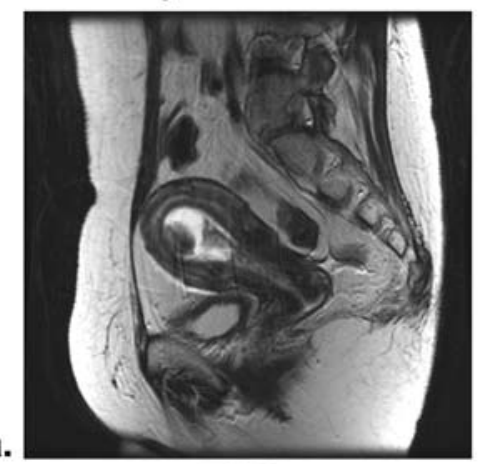

T2WI

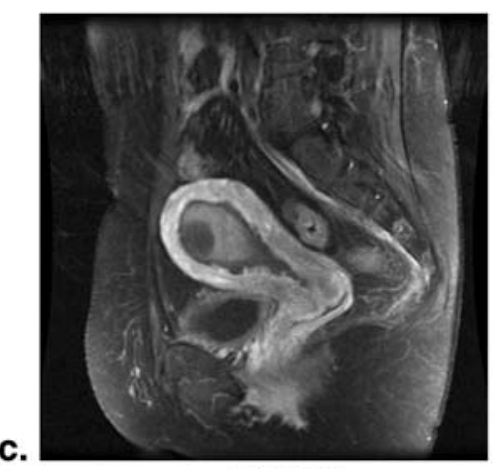

T1WI Gd
3 months after treatment

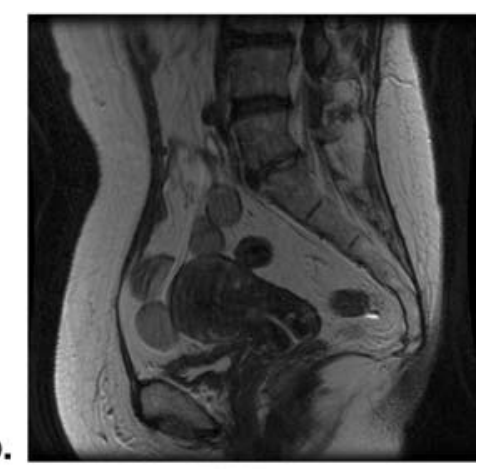

T2WI

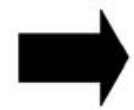

d.

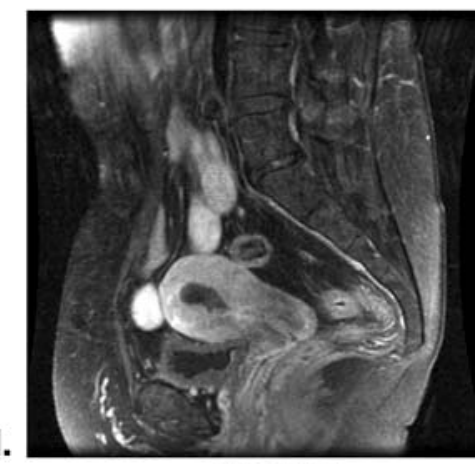

T1WI Gd 
ablation as a way to manage erratic perimenopausal bleeding. The fertility after endometrial destruction is low and is reported to be $0.7 \%$, although there is a report of pregnancy after MEA [6]. Thus women considering this procedure should be carefully counseled that though pregnancy following ablation is possible, desired fertility is a contraindication given that EA can significantly increase the risk of obstetric complications mainly due to the development of uterine synechiae.

Microwave ablation therapy has been extensively utilized in the liver and kidney. The use of microwaves at $9.2 \mathrm{GHz}$ in gynecology was first reported in the form of MEA at the University of Bath [7]. This particular device, however, was unsuitable for treating uterine cavities remarkably distorted or enlarged by uterine myomas. Recently, Kanaoka et al. [4] developed a special instrument which delivers $2.45-\mathrm{GHz}$ microwaves through a thin curved microwave applicator that conforms to the curvature of the uterine cavity. This applicator may be used in cavities up to $16 \mathrm{~cm}$ in length and can treat myomas bigger than $3 \mathrm{~cm}$ [4].

MEA has been evaluated extensively in randomized trials against first- and second-generation endometrial ablative techniques and has been shown to have good outcomes with a high level of patient satisfaction [1]. We used the device modified by Kanaoka et al. [4]. In the case of our first patient, a poor surgical candidate, the size of her myoma exceeded that which could be treated with a conventional MEA device. In the case of the second patient, MEA was useful emergently to control a life-threatening hemorrhage. Neither of the patients experienced any significant intra- or post-operative complications related to the procedure. Moreover, menorrhagia was successfully controlled and subsequent cycles were normal. MEA therefore is a promising new method for controlling life-threatening uterine bleeding in patients who are poor surgical candidates.

\section{Conflict of interest statement None.}

Open Access This article is distributed under the terms of the Creative Commons Attribution Noncommercial License which permits any noncommercial use, distribution, and reproduction in any medium, provided the original author(s) and source are credited.

\section{References}

1. Sambrook A, Cooper KG (2007) Second-generation treatment: microwave. Best Pract Res Clin Obstet Gynaecol 21:969-977. doi:10.1016/j.bpobgyn.2007.03.016

2. Qian Y, Gan N, Zhou J, Lu W, Ma Y, Zhang W (2005) Microwave endometrial ablation for menorrhagia in patients with systemic disorders. Int J Gynaecol Obstet 91:32-35. doi:10.1016/j.ijgo.2005.06.002

3. Cooper KG, Bain C, Lawrie L, Parkin DE (2005) A randomised comparison of microwave endometrial ablation with transcervical resection of the endometrium; follow-up at a minimum of five years. BJOG 112:470-475. doi:10.1111/j.1471-0528.2004.00511.x

4. Kanaoka Y, Hirai K, Ishiko O (2005) Microwave endometrial ablation for menorrhagia caused by large submucous myomas. J Obstet Gynaecol Res 31:565-570. doi:10.1111/j.1447-0756.2005.00338.x

5. Tawfeek S, Sharp N (2006) Successful microwave endometrial ablation (MEA) in uterus didelphys. J Obstet Gynaecol 26(6):582583. doi:10.1080/01443610600830979

6. Lo JS, Pickersgill A (2006) Pregnancy after endometrial ablation: English literature review and case report. J Minim Invasive Gynecol 13:88-91. doi:10.1016/j.jmig.2005.12.006

7. Sharp NC, Cronin N, Feldberg I, Evans M, Hodgson D, Ellis S (1995) Microwaves for menorrhagia: a new fast technique for endometrial ablation. Lancet 346:1003-1004. doi:10.1016/S01406736(95)91689-x 